OPEN ACCESS

Edited by:

Gong Cheng,

Tsinghua University, China

Reviewed by:

Long Yang,

New York Medical College, USA

Qiangming Sun,

Chinese Academy of Medical

Sciences, China

*Correspondence:

Renli Zhang

renlizhangszcdc@aliyun.com

Received: 18 February 2017 Accepted: 12 April 2017 Published: 09 May 2017

Citation:

Wang $M$, Yang $F$, Huang $D$, Huang $Y$,

Zhang $X$, Wang $C$, Zhang $S$ and

Zhang R (2017) Anti-Idiotypic

Antibodies Specific to prM

Monoantibody Prevent Antibody Dependent Enhancement of Dengue Virus Infection.

Front. Cell. Infect. Microbiol. 7:157. doi: 10.3389/fcimb.2017.00157

\section{Anti-Idiotypic Antibodies Specific to prM Monoantibody Prevent Antibody Dependent Enhancement of Dengue Virus Infection}

\author{
Miao Wang ${ }^{1,2}$, Fan Yang ${ }^{2}$, Dana Huang ${ }^{2}$, Yalan Huang ${ }^{2}$, Xiaomin Zhang $^{2}$, Chao Wang ${ }^{2}$, \\ Shaohua Zhang ${ }^{2}$ and Renli Zhang ${ }^{1,2 *}$ \\ ${ }^{1}$ College of Life Science and Oceanography, Shenzhen University, Shenzhen, China, ${ }^{2}$ Shenzhen Center for Disease Control \\ and Prevention, Shenzhen, China
}

Dengue virus (DENV) co-circulates as four serotypes (DENV1-4). Primary infection only leads to self-limited dengue fever. But secondary infection with another serotype carries a higher risk of increased disease severity, causing life-threatening dengue hemorrhagic fever/dengue shock syndrome (DHF/DSS). Serotype cross-reactive antibodies facilitate DENV infection in Fc-receptor-bearing cells by promoting virus entry via Fc $\gamma$ receptors $(F c \gamma R)$, a process known as antibody dependent enhancement (ADE). Most studies suggested that enhancing antibodies were mainly specific to the structural premembrane protein (prM) of DENV. However, there is still no effective drugs or vaccines to prevent ADE. In this study, we firstly confirmed that both DENV-2 infected human sera (anti-DENV-2) and DENV-2 prM monoclonal antibody (prM mAb) could significantly enhance DENV-1 infection in K562 cells. Then we developed anti-idiotypic antibodies (prM-AIDs) specific to prM mAb by immunizing of Balb/c mice. Results showed that these polyclonal antibodies can dramatically reduce ADE phenomenon of DENV-1 infection in K562 cells. To further confirm the anti-ADE effect of prM-AIDs in vivo, interferon- $\alpha$ and $\gamma$ receptor-deficient mice (AG6) were used as the mouse model for DENV infection. We found that administration of DENV-2 prM mAb indeed caused a higher DENV-1 titer as well as interleukin-10 (IL-10) and alaninea minotransferase (ALT) in mice infected with DENV-1, similar to clinical ADE symptoms. But when we supplemented prM-AIDs to DENV-1 challenged AG6 mice, the viral titer, IL-10 and ALT were obviously decreased to the negative control level. Of note, the number of platelets in peripheral blood of prM-AIDs group were significantly increased at day 3 post infection with DENV-1 compared that of prM-mAb group. These results confirmed that our prM-AIDs could prevent ADE not only in vitro but also in vivo, suggested that anti-idiotypic antibodies might be a new choice to be considered to treat DENV infection.

Keywords: dengue virus, prM antibody, antibody-dependent enhancement, anti-idiotypic antibodies, in vitro and in vivo 


\section{INTRODUCTION}

Dengue virus (DENV) is a mosquito-borne virus circulating with four distinct, but closely related serotypes (DENV1-4). The virus is transmitted to humans through bites of infected Aedes albopictus or Aedes aegypti. The incidence of dengue has grown dramatically around the world in recent decades. One estimate indicates 390 million dengue infections per year, of which 96 million clinically apparent cases and 3.9 billion people are at risk of infection in 128 countries (Brady et al., 2012; Bhatt et al., 2013).

Most people who are infected with DENV only show symptoms of mild dengue fever, but some may progress to severe dengue hemorrhagic fever (DHF) or dengue shock syndrome (DSS). Infection by one serotype provides lifelong immunity against that particular serotype (Reich et al., 2013; Forshey et al., 2016). However, subsequent infection by another serotype may increase the risk of developing severe dengue (Sangkawibha et al., 1984; Guzman et al., 2000; Screaton et al., 2015). It has been proven that infants born to dengue immunized mothers have higher risk of DHF during primary infection with DENV (Chau et al., 2008; Clapham et al., 2015). One explanation of severe DENV infections is the theory of antibody dependent enhancement (ADE) raised by Halstead in 1977 (Halstead and O'Rourke, 1977). The enhancing antibodies facilitate virus entry into susceptible myeloid cell types via FcR pathway and trigger the massive release of inflammatory and vasoactive mediators, which contribute to the disease severity (Halstead and O'Rourke, 1977; Flipse et al., 2013). High viral load, liver injury and vascular leakage are the cardinal features of severe dengue (Wichmann et al., 2004). Both sera interleukin 10 (IL-10) and alanine aminotransferase (ALT) levels were higher in those with severe dengue compared to those with mild dengue (Malavige et al., 2013; Liao et al., 2015). And high levels of IL-10 and ALT were found to associate with liver failure in dengue infections (Ferreira et al., 2015; Fernando et al., 2016). Therefore, IL-10 and ALT can be taken as the biomarkers of sever dengue disease (John et al., 2015).

Dengue virus (DENV) contains 180 copies of envelop (E) and membrane (M) protein. The premembrane (prM) protein, which consists of two moieties of the $\mathrm{pr}$ and $\mathrm{M}$ domins, is the precursor of $M$ protein. During virus maturation, furin protein cleaves prM protein to $\mathrm{M}$ protein in the trans-Golgi compartment. However, about $30 \%$ immature virions are released from infected cells (Zybert et al., 2008). A study reported that prM antibodies of DENV-2 facilitate efficient binding and cell entry of the immature type 2 viral particles into Fc-receptor-expressing cells (Rodenhuis-Zybert et al., 2010). The immune response in human to DENV is mainly caused by prM antibodies, which are highly cross-reactive among the four DENV serotypes (Beltramello et al., 2010; Dejnirattisai et al., 2010; Flipse and Smit, 2015). They do not neutralize infection but potently promote $\mathrm{ADE}$ even at high concentrations (Beltramello et al., 2010; Dejnirattisai et al., 2010). Therefore, it is the major challenge to design effective vaccine without triggering $\mathrm{ADE}$ activities because most current vaccines containing naive dengue prM protein (Ramakrishnan et al., 2015).
The lack of suitable animal models hinders the development of anti-dengue drugs. Currently, there is no specific treatment for severe dengue disease. It is urgent to search novel therapeutic strategies to block ADE. Anti-idiotypic antibodies (Anti-ids) are antibodies against the antigen binding sites of another antibody. Anti-ids have been studied and used in a variety of situations including therapeutic agents (Pan et al., 1995). Thus, we firstly developed mouse polyclonal anti-ids (prM-AIDs) targeting the antigen binding sites of DENV-2 prM monoclonal antibody (prM $\mathrm{mAb}$ ). Then we displayed the anti-ADE effects of the prM-AIDs in both $\mathrm{K} 562$ cells and a recently reported IFN $-\alpha$ and $-\gamma$ receptors deficient mouse model (C57BL/6 strain, AG6 strain) for DENV infection (Liu et al., 2016). Our results suggested that anti-ids specific to prM antibody might be considered as a potential candidate to reduce ADE in DENV infection.

\section{RESULT}

\section{ADE of DENV Infection Mediated by Human Anti-Denv-2 Sera in K562 Cells}

To confirm whether anti-DENV-2 antibodies could induce ADE to DENV-1 infection, we collected six DENV-2 infected human sera ( $\alpha$-DENV-2). Dengue IgG in the sera was detected by ELISA. Results showed five of them were considered positive of dengue IgG. The average optical density values (O.D.) of DENV IgG in $\alpha$-DENV-2 and control group were $0.439 \pm 0.278$ and 0.137 \pm 0.056 , respectively (Figure 1A). To confirm whether proteins of DENV-1 cross-reactive with human anti-DENV-2 sera and a commercial mouse DENV-2 prM mAb, we used DENV-1 as antigens and the mixed sera of six patients or prM $\mathrm{mAb}$ as the first antibody for western blot. Results showed human anti-DENV2 sera contained corresponding anbodies for prM, M, NS1 and E protein of DENV-1. Anti-DENV-2 prM mAb could react with DENV-1 prM protein (Figure 1B).

Dengue virus (DENV) mainly infected monocytes, macrophages and dendritic cells in patients (Jessie et al., 2004). Human monocyte cell line K562 (Fc receptor-bearing) was a common cell type to demonstrate the ADE of DENV in vitro. To confirm whether the human anti-DENV-2 sera could induce ADE, we infected K562 cells with DENV-1, then determined the viral load in the supernatant by qRT-PCR and detected infected cell number by flow cytometry. PCR results showed 10-100 fold enhancement of infection in four human anti-DENV-2 sera (Figure 1C). Similarly, anti-DENV-2 sera also led to a significant increase of infected cells (Figure 1D).

\section{ADE of DENV Infection Mediated by prM mAb in K562 Cells}

Next, to determine ADE effect of mouse prM mAb, we incubated K562 cells with DENV-1 $(\mathrm{MOI}=1)$ and a series of diluted prM mAb for $72 \mathrm{~h}$. Results showed that prM mAb had strong enhancement effect for DENV-1 infection in a concentration-dependent manner. A 100 -fold enhancement of DENV replication was detected with $0.1,0.25$ and $0.5 \mu \mathrm{g} / \mathrm{ml}$ prM mAb when analyzed by qRT-PCR (Figure 2A). Less than one percent of monocytes were infected with DENV-1 without 

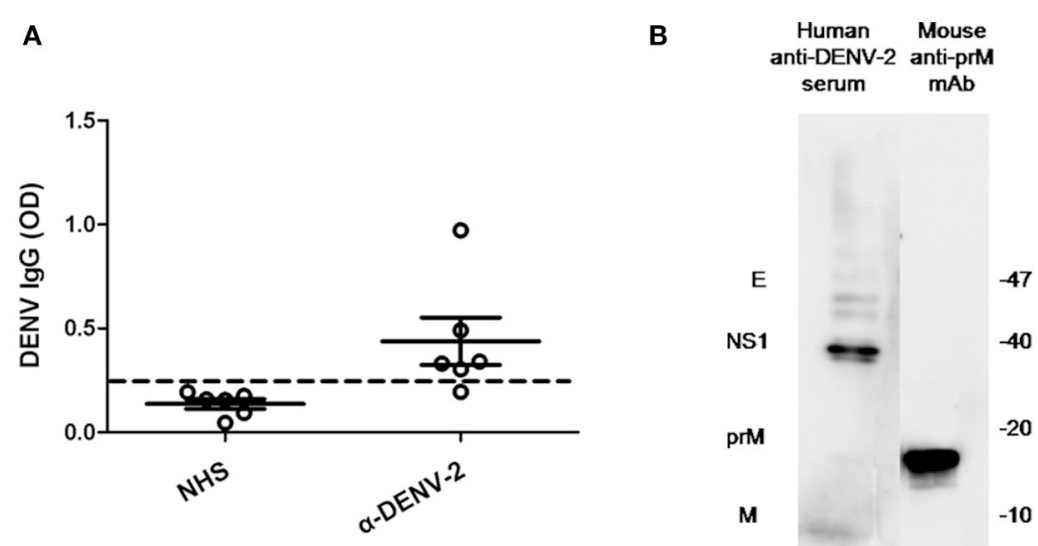

C

D
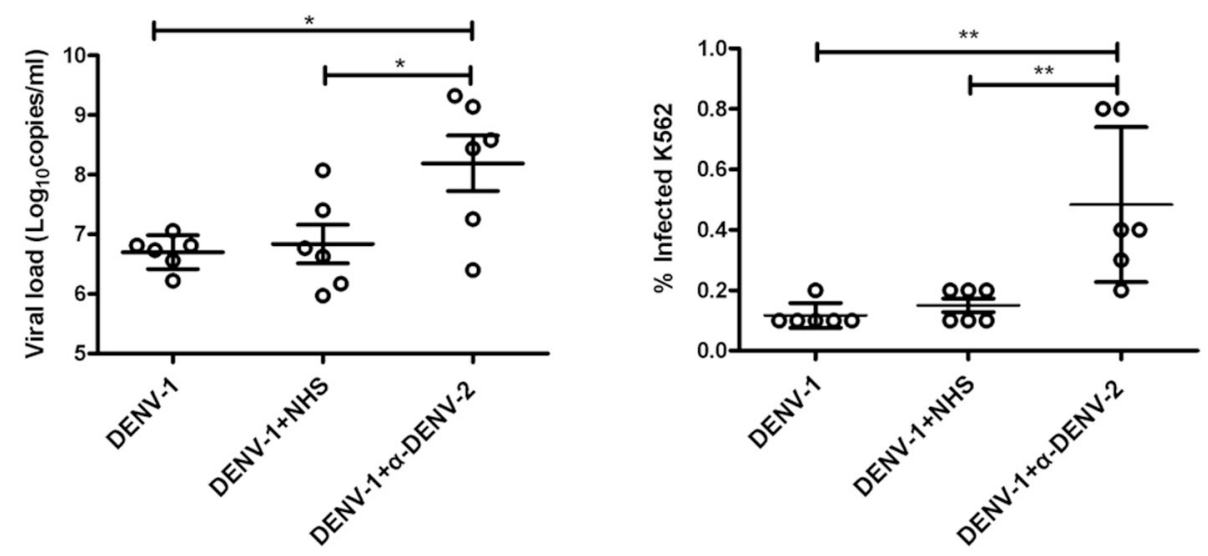

FIGURE 1 | Cross-reactivity and enhancing properties of human anti-DENV-2 sera. (A) The OD of DENV IgG in the naive human sera (NHS) and human anti-DENV-2 sera ( $\alpha$-DENV-2) was determined by ELISA $(n=6)$. (B) Western blot of DENV-1 showing reactivity with antibodies of dengue $E$, NS1, prM and M in human anti-DENV-2 sera. (C) Human anti-DENV-2 sera was incubated with DENV-1 for $1 \mathrm{~h}$ at $25^{\circ} \mathrm{C}$, then they were transferred to $\mathrm{K} 562 \mathrm{cells}$ at MOI of 1 and kept incubation for $72 \mathrm{~h}$. Viral RNA copies of infected supernatant were quantified by qRT-PCR. (D) Infected cells were determined by flow cytometry. Error bars show the means \pm SEM and pairwise comparisons were performed by unpaired test $\left({ }^{*} p<0.05,{ }^{* *} p<0.01\right)$. Dotted line presents the limit value to be considered positive.
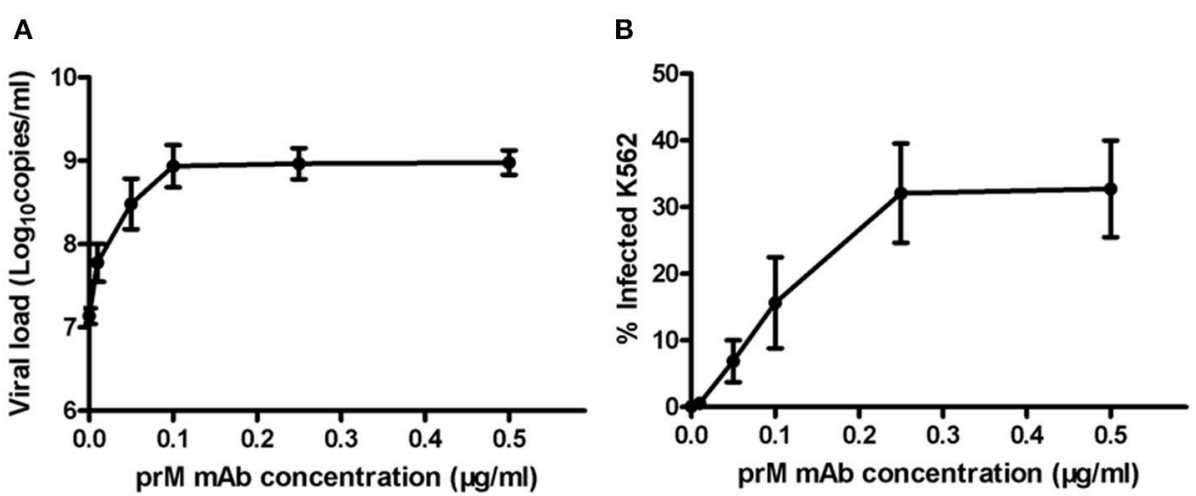

FIGURE 2 | ADE of DENV replication in K562 cells mediated by prM mAb. prM mAb was diluted to a series of different concentrations, then they were incubated with DENV-1 for $1 \mathrm{~h}$ at $25^{\circ} \mathrm{C}$ and transferred to $\mathrm{K} 562$ cells at MOI of 1 . Infected cells were incubated for $72 \mathrm{~h}$ at $37^{\circ} \mathrm{C}$. (A) Viral RNA copies in infected supernatant were quantified by qRT-PCR. (B) Infected cells were determined by flow cytometry. Data are expressed as means of three independent experiments. Error bars show the means \pm SEM. 
prM mAb. However, the percent of infected cells displayed an increased trend along with higher $\mathrm{prM} \mathrm{mAb}$ concentration. The largest DENV-1 infected cell percentage was detected when the added prM mAb concentration was increased to $0.25 \mu \mathrm{g} / \mathrm{mL}$ (Figure 2B). These results illustrated that prM mAb could enhance the infection of heterotypic virus and might played a vital role in dengue disease.

\section{Characterization of prM-AIDs}

Then, to prevent ADE induced by prM mAb, we developed anti-idiotypic antibodies (prM-AIDs) specific to prM mAb by immunizing balb/c mice. prM-AIDs in immunized mice were detected by ELISA and the titer was increased continually from day 7 after mouse immunization (Figure 3A). When the titer of anti-sera reached 1:40000 at day 35 (Figure 3A), mice were sacrificed to collect blood. prM-AIDs in the sera and naive mouse antibodies (NM-Abs) were purified by Protein-G column. The quantity and quality of purified prM-AIDs was determined by SDS-PAGE and ELISA. Results showed the purified antibody's heavy chain (about $55 \mathrm{KDa}$ ) and light chain (about $25 \mathrm{KDa}$ ) (Figure 3B). The optical density values of prM-AIDs in the naive mouse antibodies (NM-Abs) or immunized mouse antibodies (IM-Abs) and $0.093 \pm 0.014$ and $0.28 \pm 0.073$, respectively (Figure 3C). These data demonstrated balb/c mice immunized with prM mAb produced a high level of prM-AIDs.

\section{prM-AIDs Inhibited ADE of DENV Infection in K562 Cells}

To determine whether our purified prM-AIDs could inhibit ADE of DENV infection, K562 cells were infected with DENV-1 virus and prM antibody and various concentrations of prM-AIDs. prM $\mathrm{mAb}$ was added instead of prM-AIDs as a control group. The results showed that if only adding prM $\mathrm{mAb}$ and DENV-1, viral load in supernatant was significantly higher than the DENV1 alone group. But when we added prM-AIDs, the viral load was decreased in a dose-dependent manner. Compared with the prM mAb group $\left(8.2 \pm 2.8 \times 10^{8}\right.$ copies $\left./ \mathrm{mL}\right)$, the viral load in the supernatant with prM-AIDs $(25 \mu \mathrm{g} / \mathrm{mL})$ was dramatically decreased to $7.8 \pm 3.4 \times 10^{7}$ copies $/ \mathrm{mL}$ (Figure 4A). Therefore, prM-AIDs significantly diminished ADE induced by prM mAb through decreasing 90\% viral replication (Figure 4B).

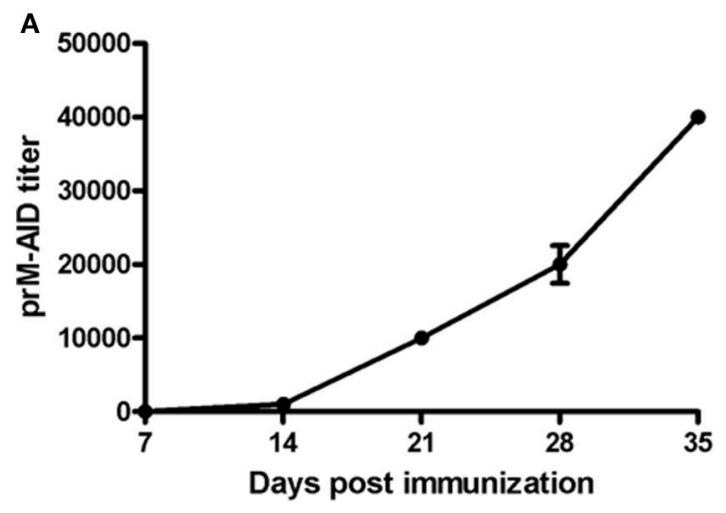

C

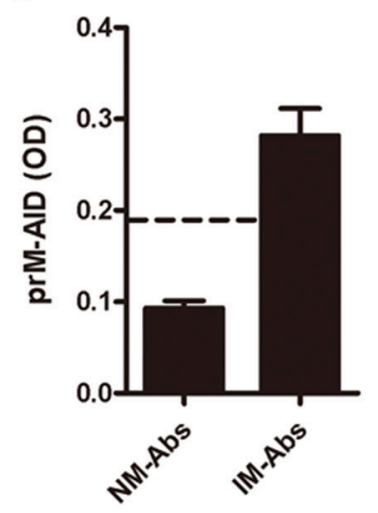

B KDa Marker prM-AID

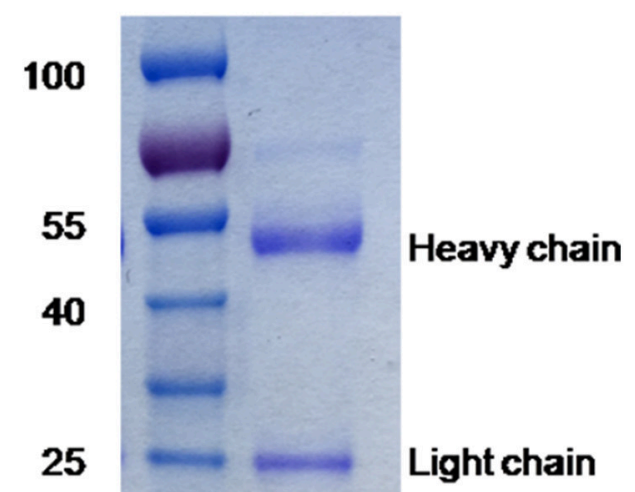

FIGURE 3 | Characterization of prM-AIDs. (A) The titer of prM-AIDs in immunizzed sera were determined by ELISA every 2 weeks $(n=6)$. Dilution of immunized or naive mouse sera were coated in 96-well plates. Biotinylated prM mAb were added. Bound prM mAb was detected by addition of streptavidin-HRP. (B) Purified prM-AIDs was identified by SDS-PAGE. (C) The specificity of prM-AIDs was analyzed by ELISA. Diluted naive mouse antibodies (NM-Abs), or immunized mouse antibodies (IM-Abs) were coated in 96-well plates. Biotinylated prM mAb were added. Bound prM mAb was detected by addition of streptavidin-HRP. Dotted line shows samples were considered positive if Positive/Native ration $>2.1$. 
A

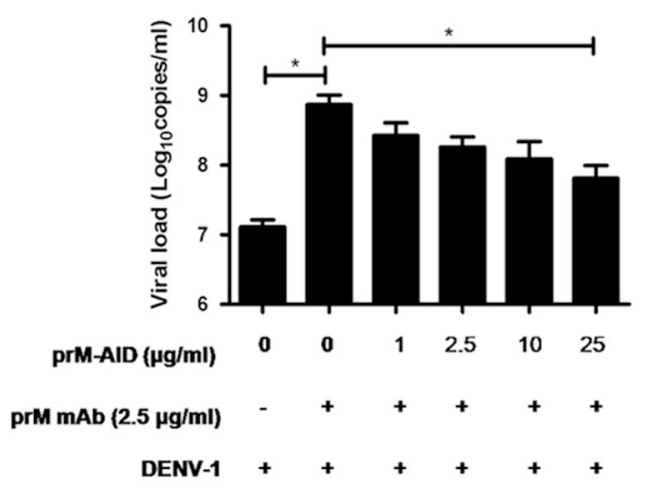

B

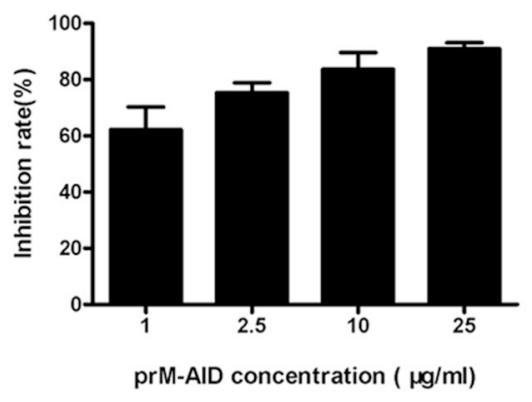

FIGURE 4 | ADE of DENV infection blocked by prM-AIDs in K562 cells. (A,B) prM-AIDs was incubated for $1 \mathrm{~h}$ at $25^{\circ} \mathrm{C}$ with $\mathrm{DENV}-1$ and $0.25 \mu \mathrm{g}$ prM mAb at an $\mathrm{MOI}$ of 1 . The mixtures infected K562 cell for $72 \mathrm{~h}$. The virus in the supernatant was determined by qRT-PCR. Data are expressed as means of three independent experiments. Error bars show the means \pm SEM and paired comparisons were performed by unpaired test $\left({ }^{*} p<0.05\right)$.

\section{ADE of DENV Infection Mediated by prM mAb in AG6 Mice}

To assess the ability of prM-AIDs to block ADE in vivo, we utilized a recently described AG6 mouse model (interferon- $\alpha$ and $\gamma$ receptor deficient mice) for ADE of dengue infection. (Figure 5A) prM $\mathrm{mAb}$ with or without prM-AIDs were transferred into AG6 mice by i.v. prior to infection with DENV1. Antibodies from naive mouse sera were purified as control antibodies (NM-Abs). Viral RNA load in blood cells was detected by qRT-PCR (Figure 5B), while infective viral particles in plasma was detected by plaque assay (Figure 5C). QA peak viremia titer in blood cells was detected at day 3 post infection (Figure 5B). Compared with the NM-Abs group, the viremia titer was 1fold higher in the prM mAb group (Figure 5B). At day 3 after inoculation, the platelet counts in DENV-1 injected mice were significantly lower than that of uninfected mice. Challenging with prM mAbs significantly reduced platelets (Figure 5D).

To further explore the inflammation response and liver damage of ADE infection, we also detected ALT (Figure 5E) and IL-10 (Figure 5F) in the plasma of mice at days 3 post infection by ELISA assay. Compared with the uninfected groups, a significant higher level of ALT and IL-10 were observed in the DENV infected groups (Figures 5E,F). Compared to the pure DENV-1 infected mice group, the level of ALT and IL-10 were increased in the prM mAb mice group. These data show that ADE of DENV-1 infection in AG6 mice by passive transfer prM antibody.

\section{prM-AIDs Inhibited ADE of DENV Infection in AG6 Mice}

However, by comparison, a significant difference in viral load of blood cell between the mice that received prM-AID and the mice that received prM $\mathrm{mAb}$ at day 3 post infection (Figure 5B). Similarly, the viral load in the plasma also showed a peak at day 3 post infection. Although the viral titer in the prM-AIDs group displayed the lowest trend, there was no statistically significant difference among all the groups. Compared with the prM mAb group, the mean viremia titer in the prM-AIDs group decreased about 1-fold (Figure 5C).

Interestingly, the number of the platelets in the prM-AIDs challenged mice was significantly higher than that of NM-Abs group at day 3 post infection (Figure 5D). Besides, challenged with prM-AIDs showed decreased trend of the level of ALT and IL-10 than the prM mAb mice group and naive mouse antibodies group (Figures 5E,F).

\section{DISCUSSION}

In this study, we firstly confirmed ADE to DENV-1 infection induced by human anti-DENV-2 sera and murine anti-DENV2 prM mAb in K562 cells. Then we developed and characterized prM-AIDs targeting the antigen-binding site of prM mAb. We showed that prM-AIDs significantly inhibited ADE mediated by prM mAb in vitro. Importantly, administration of prM-AIDs to AG6 mice largely reduced the disease severity of DENV-1 infection.

Secondary infection with another DENV serotype carries an increased risk of severe disease (Soo et al., 2016). A high viral load in blood, severe thrombocytopenia, and platelet dysfunction may result in increased capillary fragility (Ojha et al., 2017), clinically manifested as petechiae, easy bruising, and gastrointestinal mucosal bleeding (Deshwal et al., 2016), which is characteristic of DHF/DSS. ADE has been proposed as an underlying pathogenic mechanism of DHF/DSS (Halstead, 1970). On one hand, enhancing antibodies can bind DENV through Fab fragments, on the other hand, virus-antibodies complex still could recognize Fc receptor-bearing cells via their Fc region, leading to increased virus uptake and replication (Gan et al., 2017). Many evidences showed viral premembrane protein prM mAbs broad cross-activity with the four DENV serotypes and showed poor neutralization function but potently promote ADE (Beltramello et al., 2010; Dejnirattisai et al., 2010). To confirm the ADE induced by prM mAb, we incubated DENV1 infected K562 cells with either human anti-DNEV2 sera or 


\section{A}

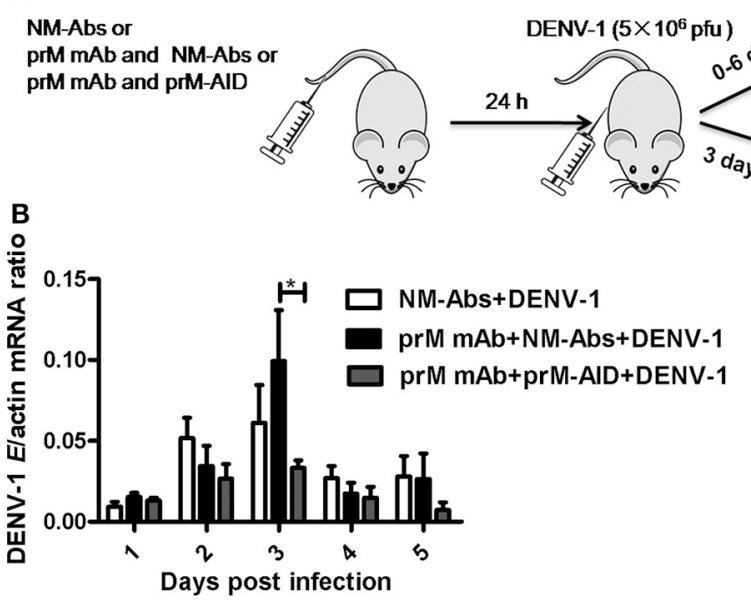

D

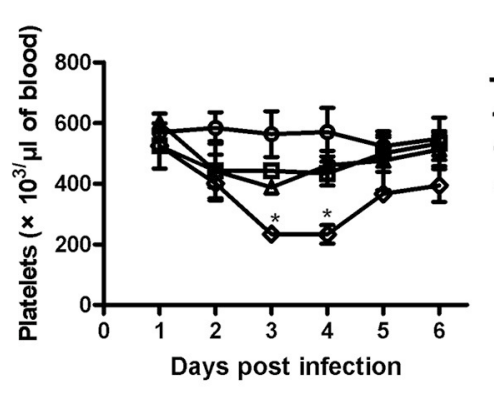

E
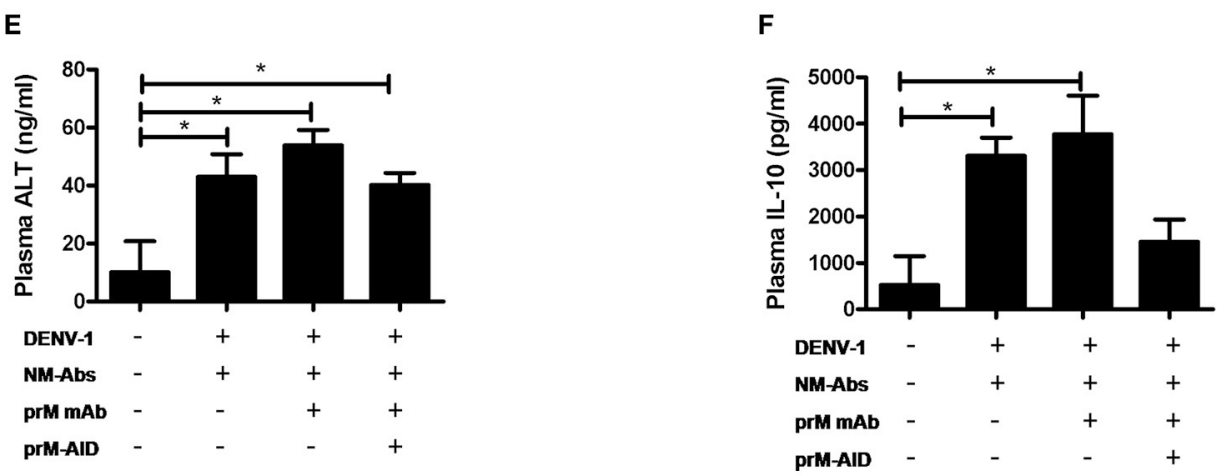

FIGURE 5 | prM-AIDs inhibited ADE of DENV infection in vivo. (A) Schematic representation of the study design. Mice were first administered with naive mouse antibodies (NM-Abs), prM mAb and prM-AIDs, then $24 \mathrm{~h}$ later they were challenged with DENV-1 by i.p.. Blood was collected from the vail veins from 0 to 5 days post-infection. (B) Viral copies in the blood cells were quantified by qRT-PCR. (C) Infectious viral particles in the plasma were assessed by plaque assay. (D) Platelets in blood were counted using a hemocytometer under a light microscope. (E,F) The levels of ALT and IL-10 in plasma were measured by ELISA at day 3 post-infection. $n=4-5$ mice per group. Error bars show the means \pm SEM and pairwise comparisons were performed by non-parametric Mann-Whitney test ( $\left.{ }^{*} p<0.05\right)$.

mouse prM mAb. The K562 cell line was chosen as a simple and easily interpretable model system for the in vitro study of $\mathrm{ADE}$ and ADE inhibition as it only expresses the Fc $\gamma \mathrm{II}$ receptor (Clark et al., 2016). ADE induced by patients' sera had once been reported in laboratory virus strains (Chaichana et al., 2014; de Alwis et al., 2014). Here, a clinical DENV-1 isolate was taken to perform ADE assay. We confirmed clinical isolate DV1 infection was enhanced by human anti-DV2 sera or murine anti-DV2 prM mAb in Fc $\gamma$ receptor-bearing K562 cells. Besides, since the clinical isolated DENV-1 virus strain and the anti-DENV-2 sera were both derived from Guangdong local DENV infected patients. It indicates that people living in Guangdong who were primarily infected with DENV-2 have high risk of developing severe dengue disease once they are further attacked by DENV-1.

To date, no entirely successful attempt of active immunization or drug treatment in the field of DENV has been published. The only licensed tetravalent dengue vaccine Dengvaxia (CYD-TDV) 
which was developed by Sanofi Pasteur and TAK-003 (Takeda) as a candidate vaccine both showed moderate efficacy against dengue infection (Osorio et al., 2014; Godoi et al., 2017). However, considering the $\mathrm{ADE}$ activities, current vaccines have a potential risk of aggravating disease (Aguiar et al., 2017). A recent report published on science suggested that vaccination of Dengvaxia in low-transmission settings might increase the incidence of more severe "secondary-like" infection and hospitalizations (Ferguson et al., 2016). Meanwhile, some anti-viral compounds have shown efficient ADE inhibition of DENV (Ayala-Nunez et al., 2013; Flingai et al., 2015). Anti$\mathrm{TNF} \alpha$ therapy totally protected the DENV-2 infected A129 mice (Martinez Gomez et al., 2016). Importantly, humanized anti-DENV antibodies engineered with mutations in Fc region were developed to prevent binding to $\mathrm{FCR}$ for the treatment of dengue disease in AG129 mice (Goncalvez et al., 2007; Balsitis et al., 2010; Beltramello et al., 2010; Ramadhany et al., 2015). A recent study reported plant-produced anti-dengue virus monoclonal antibodies exhibited reduced ADE activity in FcR expressing human cells (Dent et al., 2016). However, to our knowledge, no method mentioned above has been widely accepted. As a consequence, passive immune therapy of antiidiotypic antibodies by blocking enhancing antibodies may provide an alternative strategy for the treatment of dengue, especially for patients who have already acquired primary infection or reside in endemic areas.

When one antibody binds to the variable region of another antibody, it is referred to as an anti-idiotypic antibody. Over the past years, anti-idiotypic antibodies have been studied and used in a variety of situations including attempts to use them as therapeutic agents (Denapoli et al., 2017). They are ideal for bioanalytical assays in preclinical research and clinical drug monitoring due to their high specificity and sensitivity (Bulashev et al., 2016). One research showed that anti-idiotypic antibodies could inhibit the binding between autoantibodies and autoantigens, resulting to prevent the development of autoimmune disease (Hampe, 2012). In this study we produced high titer of prM-AIDs by immunizing mice with prM mAbs. Results confirmed that prM-AIDs significantly inhibited DENV-1 replication in K562 cells in a dose-dependent manner. Although other modified antibodies could reduce their enhancing activity (Dent et al., 2016; Wang et al., 2017), they couldn't block the enhancing antibodies that already existed in primary infection. Polyclonal antibodies can be easily raised, as their production steps do not require any sophisticated laboratory facility. Therefore, anti-idiotypic antibody therapy represents a novel approach for dengue disease prevention and treatment. They have great potential to be used in combination with viral replication inhibitors that will decrease the emergence chance of resistant virus strains.

Many ADE-related studies were limited in in vitro assays (Dent et al., 2016; Wang et al., 2017). The evidence demonstrating ADE in vivo was first described by Healstead in rhesus monkeys (Halstead, 1979). Recently, a new animal model AG6 mice (interferon- $\alpha$ and $\gamma$ receptor deficient mice) for dengue infection was reported to study the function of secreted DENV NS1 protein (Liu et al., 2016). In this model, DENV could lead to lethal disease characterized by reduced white blood cells and platelets as well as vascular leakage (Orozco et al., 2012; Liu et al., 2016), all features associated with severe dengue symptoms in humans (Halstead, 2007). Therefore, we chose AG6 mice as an animal model system for the study of $\mathrm{ADE}$ and $\mathrm{ADE}$ inhibition in vivo. ADE of DENV-1 replication in AG6 mice was demonstrated by passive antibody transfer. Increased virus titers, IL-10 and ALT in the blood were observed in the mice after prM antibody injection by i.v. Besides, the number of the platelets was 2 -fold lower in mice challenged with prM antibody than that in the control mice. These results were similar to those in another ADE mice modelAG129 (IFN- $\alpha$ and $\beta$ receptor deficient) (Balsitis et al., 2010; Martinez Gomez et al., 2016). Here, we first demonstrated ADE in AG6 mice by passively transfering prM mAb, although the enhancing effect was not very significant compared with other studies using different ADE mice models. We proposed that the different virus strains as well as viral challenging routes and doses might impact the disease progression and manifestations. For example, in this study, we challenged mice with DENV-1, which was believed to be weaker than DENV-2 used in other studies in pathogenesis.

Next, to confirm whether our prM-AIDs had the same inhibitory effect in vivo similar to that in cells, we challenged AG6 mice with both DENV-1 and prM-AIDs. Results showed prM-AIDs were capable to block the ADE effect and reducing the viral burden to an equivalent level to the DENV-1 control group in absence of prM mAb During the $\mathrm{ADE}$ and severe dengue symptoms, reduced platelets, platelet dysfunction as well as liver damages were common pathogenesis mechanisms. Therefore, in this study, besides the viral load in blood cells and the plasma, the levels of IL-10, ALT and platelet counts in the blood were also detected. We found the levels of IL-10 and ALT were decreased by prM-AIDs, suggesting that mice had reduced inflammatory responses and liver damages. The number of platelets in the peripheral blood of prM-AIDs group returned to normal level, which hinted that vascular damages and bleeding possibility were also reduced. These results demonstrated that the prM-AIDs could inhibit $\mathrm{ADE}$ induced by prM $\mathrm{mAb}$ in vivo at least from immune responses as well as liver functions and platelet counts. The mechanisms of anti-ADE by prM-AIDs might be that they specifically bound to the antigenic determinants of prM mAbs, resulting in inhibition of the binding between prM mAbs and viruses. Further studies needed to be done to confirm the detailed anti-ADE mechanisms of prM-AIDs.

Taken together, we successfully demonstrated the ADE in both cell level and in a recently reported AG6 mice model by challenging prM mAbs. Then, we developed prM-AIDs specific to this anti-dengue prM mAb. Furthermore, the prM-AIDs successfully inhibited ADE not only in vitro but also protected mice avoiding severe inflammatory responses as well as liver damages in vivo. It provided a new strategy to develop specific treatment for severe DENV infection.

\section{MATERIALS AND METHODS}

\section{Ethics Statement}

All patient samples and data were assigned institution-specific identification numbers to ensure patient anonymity.Balb/c mice were purchased from Guangdong medical Laboratory Animal 
Center. The mice were bred and maintained under SPF animal house in Shenzhen Center for Disease Control and Prevention. C57BL/6 mice deficient in type I and type II interferon (IFN) receptors (AG6 mice) were purchased from Institute Pasteur of Shanghai, Chinese Academy of Science, which is bred and maintained under SPF animal house in Tsinghua University. All experiments procedures were approved and performed according to the guidelines of the Experimental Animal Welfare and Ethics Committee of Shenzhen Center for Disease Control and Prevention.

\section{Cell Lines and Virus}

The Aedesalbopictus cell line C6/36 and Baby Hamster Kidney21 (BHK-21) cell line were cultured in Dulbecco's modification of Eagle's medium (DMEM). Human erythroleukaemic K562 cells were grown in Roswell Park Memorial Institute (RPMI) 1640 medium. All media were supplemented with $10 \%$ fetal bovine sera, $100 \mathrm{U} / \mathrm{mL}$ penicillin, and $100 \mu \mathrm{g} / \mathrm{mL}$ streptomycin. C6/36 cells were propagated at $28^{\circ} \mathrm{C}$ and BHK-21 and $\mathrm{K} 562$ cells were propagated at $37^{\circ} \mathrm{C}$. All the materials used for cell culture were purchased from GIBOCO and cells were from China Center for Type Culture Collection.

DENV-1 was isolated in our laboratory from a patient during a DENV outbreak in Shenzhen, China in 2014. Virus was propagated in C6/36 cell line. After 3-4 days, the supernatant was harvested, and cell debrises were removed by centrifuge at 10000 rpm for $10 \mathrm{~min}$ at $4^{\circ} \mathrm{C}$. DENV-1 was used after being passaged for 3 times in C6/36 cells. To increase the virus titer, the supernatant were concentrated by centrifuge at $4,000 \mathrm{~g}$ for $30 \mathrm{~min}$ at $4^{\circ} \mathrm{C}$ using filtration device (Millipore, Cat. No\# UFC901008). Virus stocks were stored at $-80^{\circ} \mathrm{C}$.

\section{Anti-DENV-2 Sera}

Human anti-DENV-2 sera samples were obtained from patients during a DENV outbreak in Guangdong, China. Human antiDENV-2 sera samples and native human sera samples were collected from Shenzhen Center for Disease Control and Prevention.

\section{Anti-prM Monoclonal Antibody}

Dengue virus (DENV) type 2 prM IgG1 monoclonal antibody, directed against pre-membrane glycoprotein of DENV serotype 2 was purchased from Thermo (Cat. No\#, MA1-71252). To remove sodium azide in the supernatant, the antibody was further purified by the Montage Prosep-G kit (Millipore, Cat. No\# P36491) and quantitated by the BCA Protein Quantitate Kit (Thermo Scientific, Cat.No\# 23225) ccording to manufacturer's instructions.

\section{Plaque Forming Assay}

The titer of virus was determined by plaque assay in BHK21 cells. BHK-21 cells were seeded in 6-well plate in DMEM with $10 \% \mathrm{FBS}$ at $37^{\circ} \mathrm{C}$. Next day, virus was serially diluted with DMEM medium and incubated with BHK cells for $1 \mathrm{~h}$. Subsequently, the viral suspension was removed, and DMEM medium containing $2 \%$ FBS and $1 \%$ low-melting point agarose were added. After incubation at $37^{\circ} \mathrm{C}$ for 6 days, cells were fixed with $4 \%$ formaldehyde for $2 \mathrm{~h}$. After the overlay was removed, cells were stained by crystal violet for a few minutes and washed carefully by water. Plaques were calculated by naked eyes.

\section{Cross-Reactivity Analysis between DENV-1 and Human Anti-DENV Sera or prM mAb by Western Blot}

The DENV-1 supernatant was electrophoresed through $12 \%$ SDS/polyacrylamide gels and then electrotransferred to nitrocellulose membrane (Millipore). The membrane was blocked with 5\% BSA in TBST before incubation overnight with human anti-DENV sera (1:100 diluted) or prM mAb at $4^{\circ} \mathrm{C}$. Wash the membrane with TBST four times. PeroxidaseLabeled Antibody to Human IgG (KPL, Cat. No\# 04-10-20, 1:2,000 dilute) or Peroxidase-Labeled Antibody to mice IgG (Abcam,Cat. No\#ab6789, 1:100,000 dilute) was added in TBST with $5 \%$ BSA for $2 \mathrm{~h}$ at room temperature. After washing the membrane, bands were detected by using SuperSignal West Pico chemiluminescent substrate (Thermo Scientific, Cat. No\#HF104626).

\section{Analysis of ADE Induced by Human Sera or prM mAb in K562 Cell}

Human sera or prM mAb was incubated with DENV-1 at an MOI of 1 for $1 \mathrm{~h}$ at $25^{\circ} \mathrm{C}$ in shaking table at 80 $\mathrm{rpm}$ to form virus-antibody complexes. The mixtures infected K562 cells in 24 -well plates $\left(1 \times 10^{5}\right.$ cells/well $)$ at $37^{\circ} \mathrm{C}$ for $72 \mathrm{~h}$. The number of infected cells was determined by flow cytometry. The virus in the supernatant was determined by qRT-PCR.

DENV-1 infected and mock K562 cells were harvested and washed with cold phosphate buffered saline (PBS). To block cell surface FcR, incubate cells with $0.5 \mu \mathrm{g}$ Human BD Fc Block $^{\mathrm{TM}}$ (BD, Cat. No\#564220) for $20 \mathrm{~min}$ at room temperature. Fixation, permeabilized and intracellular fluorescence labeling were performed by Fixation/Permeabilization solution (BD, Cat. No\#554714) according to manufacturer's instructions. For labeling, cells were incubated with anti-dengue virus $\mathrm{E}$ glycoprotein antibody (abcam, Cat. No\# ab41329, 1:20 dilute) and then labeled with Donkey F(ab')2 Anti-Mouse IgG H\&L (Alexa Fluor ${ }^{\circledR}$ 488,abcam, Cat. No\#ab181289). Finally, cells were suspended in PBS containing 1\% FBS and subjected to flow cytometry.

RNA in the supernatant was extracted using Viral RNA kit (Roche, Cat. No\#13438700). qRT-PCR was carried out with DENV General-type Real Time RT-PCR Kit (Shanghai ZJ, Cat. No\#ER-0101-02). Amplification conditions were $45^{\circ} \mathrm{C}$ for $10 \mathrm{~min}, 95^{\circ} \mathrm{C}$ for $15 \mathrm{~min}$ and 40 cycles of $95^{\circ} \mathrm{C}$ for $15 \mathrm{~s}, 60^{\circ} \mathrm{C}$ for $1 \mathrm{~min}$.

\section{Preparation of prM-AIDs}

To obtain prM-AID, six female Balb/c mice (6 weeks of age) were subcutaneously immunized with dengue prM $\mathrm{mAb}$ three times at 2-week intervals in Freund's complete(sigma, Cat. No\#F5881) or incomplete adjuvant (sigma, Cat. No\#F5506). Blood samples were collected from the tail 
vein of immunized mice to determinate the titer of antibody. Seven days after the final immunization, mice were sacrificed to take the blood. The sera were separated from the blood by centrifuge at $2,500 \mathrm{~g}$ for $30 \mathrm{~min}$ at $4^{\circ} \mathrm{C}$ and the products was purified from sera using the Montage Prosep-G kit (Millipore Cat. No\#P36491). The polyclonal antibodies were concentrated using centrifugal filter device (Millipore) and quantitated by the BCA Protein Quantitate Kit (Thermo, Cat. No\#23225) according to manufacturer's instructions. The purity of polyclonal antibodies was identified by sodium dodecyl sulfate-poly-acrylamide gel electrophoresis (SDSPAGE) on $12 \%$ polyacrylamide gels under denaturating and reducing conditions and evaluated by Coomassie blue staining.

\section{Detection of prM-AIDs by ELISA}

To dectect prM-AIDs titer in the immunized sera, serially diluted control and immunized sera were coated in 96-well plates at $4^{\circ} \mathrm{C}$ overnight. And to identify prM-AIDs in purify antibodies, antibodies purified from naive mice as negative control, and purify anti-idiotypic antibodies from immunized mice were coated in 96-well plates at $4{ }^{\circ} \mathrm{C}$ overnight. The plates were blocked by $2 \%$ BSA in PBST for $1 \mathrm{~h}$ at room temperature. The prM $\mathrm{mAb}$ was biotinylated by using NH2-reactive biotin (Elabscience, Cat. No\#EBLK0002). After washing with TBST, biotinylated prM mAb was added and incubated for $1 \mathrm{~h}$ at $37^{\circ} \mathrm{C}$. Plates were washed four times with TBST. Streptavidin-HRP (1:10,000 diluted, Beyotime, Cat. No\#A0303) were added and incubated for $30 \mathrm{~h}$ at $37^{\circ} \mathrm{C}$. Wash the plates as above. TMB was added as the substrate, and the reaction was stopped by $\mathrm{H}_{2} \mathrm{SO}_{4}$ stop solution. The OD at $450 \mathrm{~nm}$ was measured with an iMarkTM Microplate Reader. Samples were considered positive if $\mathrm{P} / \mathrm{N}$ ration $>2.1$.

\section{ADE Inhibition Assay by prM-AID in K562 Cells}

prM-AIDs was incubated for $1 \mathrm{~h}$ at $25^{\circ} \mathrm{C}$ in shaking table at $80 \mathrm{rpm}$ with DENV-1 and prM mAb at an MOI of 1 . The mixtures infected $\mathrm{K} 562$ cells in 24 -well plates at $37^{\circ} \mathrm{C}$ with $5 \%$ $\mathrm{CO}_{2}$ for $72 \mathrm{~h}$. The virus in the supernatant was determined by qRT-PCR.

\section{ADE Inhibition by prM-AID in AG6 Mice}

Twenty six to eight week-old AG6 mice were randomly into four groups. Antibodies were purified from naive mouse sera (NM-Abs) as control antibodies. Mice were injected $100 \mu \mathrm{g}$ NM-Abs, $20 \mu \mathrm{g}$ prM mAb and $100 \mu \mathrm{g}$ NM-Abs or $20 \mu \mathrm{g}$ prM $\mathrm{mAb}$ and $100 \mu \mathrm{g}$ prM-AID by intravenous injection (i.v) into the tail vein then intraperitoneal (i.p) infected $24 \mathrm{~h}$ later with $10^{6} \mathrm{PFU}$ of DENV-1. Blood samples from each mice were collected from the tail vein daily for the next 5 days. Viremia titers were detected from day 1 to day 5 post infection. Platelets were count from day 1 to day 5 post infection. The level of IL-10 and ALT were determined at day 3 post infection.

\section{Quantitative Analysis of DENV-1 Viremia in AG6 Mice}

$10 \mu \mathrm{l}$ whole blood was collected each day after viral challenge into tubes containing sodium citrate. Samples were centrifuged at $6,000 \mathrm{~g}$ and $4^{\circ} \mathrm{C}$ for $5 \mathrm{~min}$ to separate plasma and blood cells. Viral load in the plasma was determined by plaque. RNA was extracted from blood cells using an RNA Mini kit (Qiagen, Cat. No\#154025157) and reverse transcripted into cDNA using an iScript cDNA kit (Bio-Rad, Cat. No\#1708891). Viral genomes were quantified via TaqMan qPCR amplification. The DENV1 primer pairs were GCTCCCACGTCGGAAATACA and TGT TCTAGGTGAGCAGTCCAATG. The probe was FAM 5'CTG ACTGACTACGGAGCC-3' TAMRA. The sequence of mouse actin primer pairs were AGCCATGTACGTAGCCATCCA and TCTCCGGAGTCCATCACAATG. The probe was FAM 5'TGTCCCTGTATGCCTCTGGTCGTACCAC-3' TAMRA. Gene quantities were normalized against mouse actin.

\section{Detection of IL-10 and ALT by ELISA}

IL-10 (Abcam,Cat. No\#ab46103) and ALT (Cloud-clone, Cat. No\#SEA207Mu) in the infected and mock-infected mice was quantified using commercially available ELISA kits. The experiment was performed according to manufacturer's instructions.

\section{Statistical Analysis}

Unpaired $t$-test was used to comparison of viral road and percentage of infection in $\mathrm{ADE}$ and $\mathrm{ADE}$ inhibition assay. Non-parametric Mann-Whitney test were used for pairwise comparisons of viral road, IL-10, ALT and platelets in AG6 mice. All calculations were performed in GraphPad Prism 5.0 software.

\section{AUTHOR CONTRIBUTIONS}

RZ designed the experiments; RZ, MW, XZ, and $\mathrm{YH}$ wrote the manuscript and performed the majority of the experiments and analyzed data; FY, DH, CW, and SZ supplied material and performed a part of the experiments. All authors reviewed, critiqued, and provided comments to the text.

\section{FUNDING}

This work was financially supported by the National major science and technology research projects of China (No.2016YFC1202001) and science and technology research projects of Shenzhen (No. JCYJ20160427151920801).

\section{ACKNOWLEDGMENTS}

We are grateful to Prof. Gong Cheng from Tsinghua University for providing AG6 mice. We thank the supports of Shenzhen San-Ming project for prevention and Resaearch on Vector-borne Disease. 


\section{REFERENCES}

Aguiar, M., Halstead, S. B., and Stollenwerk, N. (2017). Consider stopping dengvaxia administration without immunological screening. Expert Rev. Vaccines 16, 301-302. doi: 10.1080/14760584.2017.1276831

Ayala-Nunez, N. V., Jarupathirun, P., Kaptein, S. J., Neyts, J., and Smit, J. M. (2013). Antibody-dependent enhancement of dengue virus infection is inhibited by SA-17, a doxorubicin derivative. Antiviral Res. 100, 238-245. doi: 10.1016/j.antiviral.2013.08.013

Balsitis, S. J., Williams, K. L., Lachica, R., Flores, D., Kyle, J. L., Mehlhop, E., et al. (2010). Lethal antibody enhancement of dengue disease in mice is prevented by Fc modification. PLoS Pathog. 6:e1000790. doi: 10.1371/journal.ppat.1000790

Beltramello, M., Williams, K. L., Simmons, C. P., Macagno, A., Simonelli, L., Quyen, N. T., et al. (2010). The human immune response to Dengue virus is dominated by highly cross-reactive antibodies endowed with neutralizing and enhancing activity. Cell Host Microbe 8, 271-283. doi: 10.1016/j.chom.2010.08.007

Bhatt, S., Gething, P. W., Brady, O. J., Messina, J. P., Farlow, A. W., Moyes, C. L., et al. (2013). The global distribution and burden of dengue. Nature 496, 504-507. doi: 10.1038/nature 12060

Brady, O. J., Gething, P. W., Bhatt, S., Messina, J. P., Brownstein, J. S., Hoen, A. G., et al. (2012). Refining the global spatial limits of dengue virus transmission by evidence-based consensus. PLoS Negl. Trop. Dis. 6:e1760. doi: 10.1371/journal.pntd.0001760

Bulashev, A. K., Borovikov, S. N., Serikova, S. S., Suranshiev, Z. A., Kiyan, V. S., and Eskendirova, S. Z. (2016). Development of an ELISA using antiidiotypic antibody for diagnosis of opisthorchiasis. Folia Parasitol. 63:205. doi: $10.14411 /$ fp. 2016.025

Chaichana, P., Okabayashi, T., Puiprom, O., Sasayama, M., Sasaki, T., Yamashita, A., et al. (2014). Low levels of antibody-dependent enhancement in vitro using viruses and plasma from dengue patients. PLoS ONE 9:e92173. doi: 10.1371/journal.pone.0092173

Chau, T. N., Quyen, N. T., Thuy, T. T., Tuan, N. M., Hoang, D. M., Dung, N. T., et al. (2008). Dengue in Vietnamese infants-results of infectionenhancement assays correlate with age-related disease epidemiology, and cellular immune responses correlate with disease severity. J. Infect. Dis. 198, 516-524. doi: 10.1086/590117

Clapham, H., Cummings, D. A., Nisalak, A., Kalayanarooj, S., Thaisomboonsuk, B., Klungthong, C., et al. (2015). Epidemiology of infant dengue cases illuminates serotype-specificity in the interaction between immunity and disease, and changes in transmission dynamics. PLoS Negl. Trop. Dis. 9:e0004262. doi: 10.1371/journal.pntd.0004262

Clark, K. B., Hsiao, H. M., Bassit, L., Crowe, J. E. Jr., Schinazi, R. F., Perng, G. C., et al. (2016). Characterization of dengue virus 2 growth in megakaryocyte-erythrocyte progenitor cells. Virology 493, 162-172. doi: 10.1016/j.virol.2016.03.024

de Alwis, R., Williams, K. L., Schmid, M. A., Lai, C. Y., Patel, B., Smith, S. A., et al. (2014). Dengue viruses are enhanced by distinct populations of serotype cross-reactive antibodies in human immune sera. PLoS Pathog. 10:e1004386. doi: 10.1371/journal.ppat.1004386

Dejnirattisai, W., Jumnainsong, A., Onsirisakul, N., Fitton, P., Vasanawathana, S., Limpitikul, W., et al. (2010). Cross-reacting antibodies enhance dengue virus infection in humans. Science 328, 745-748. doi: 10.1126/science.1185181

Denapoli, P. M., Zanetti, B. F., Dos Santos, A. A., de Moraes, J. Z., and Han, S. W. (2017). Preventive DNA vaccination against CEA-expressing tumors with antiidiotypic scFv6.C4 DNA in CEA-expressing transgenic mice. Cancer Immunol. Immunother. 66, 333-342. doi: 10.1007/s00262-016-1940-4

Dent, M., Hurtado, J., Paul, A. M., Sun, H., Lai, H., Yang, M., et al. (2016). Plantproduced anti-dengue virus monoclonal antibodies exhibit reduced antibodydependent enhancement of infection activity. J. Gen. Virol. 97, 3280-3290. doi: 10.1099/jgv.0.000635

Deshwal, R., Qureshi M. I., and Singh, R. (2016). Clinical and laboratory profile of dengue fever patients: a clinical experience. J. Assoc. Physicians India 64:71.

Ferguson, N. M., Rodriguez-Barraquer, I., Dorigatti, I., Mier, Y. T.-R. L., Laydon, D. J., and Cummings, D. A. (2016). Benefits and risks of the Sanofi-Pasteur dengue vaccine: modeling optimal deployment. Science 353, 1033-1036. doi: 10.1126/science.aaf9590
Fernando, S., Wijewickrama, A., Gomes, L., Punchihewa, C. T., Madusanka, S. D., Dissanayake, H., et al. (2016). Patterns and causes of liver involvement in acute dengue infection. BMC Infect. Dis. 16:319. doi: 10.1186/s12879-016-1656-2

Ferreira, R. A., de Oliveira, S. A., Gandini, M., Ferreira Lda, C., Correa, G., Abiraude, F. M., et al. (2015). Circulating cytokines and chemokines associated with plasma leakage and hepatic dysfunction in Brazilian children with dengue fever. Acta Trop. 149, 138-147. doi: 10.1016/j.actatropica.2015.04.023

Flingai, S., Plummer, E. M., Patel, A., Shresta, S., Mendoza, J. M., Broderick, K. E., et al. (2015). Protection against dengue disease by synthetic nucleic acid antibody prophylaxis/immunotherapy. Sci. Rep. 5:12616. doi: $10.1038 /$ srep 12616

Flipse, J., and Smit, J. M. (2015). The Complexity of a Dengue vaccine: a review of the human antibody response. PLoS Negl. Trop. Dis. 9:e0003749. doi: 10.1371/journal.pntd.0003749

Flipse, J., Wilschut, J., and Smit, J. M. (2013). Molecular mechanisms involved in antibody-dependent enhancement of dengue virus infection in humans. Traffic 14, 25-35. doi: $10.1111 /$ tra. 12012

Forshey, B. M., Stoddard, S. T., and Morrison, A. C. (2016). Dengue viruses and lifelong immunity: reevaluating the conventional wisdom. J. Infect. Dis. 214, 979-981. doi: 10.1093/infdis/jiw102

Gan, E. S., Ting, D. H., and Chan, K. R. (2017). The mechanistic role of antibodies to dengue virus in protection and disease pathogenesis. Expert Rev. Anti Infect. Ther. 15, 111-119. doi: 10.1080/14787210.2017.1254550

Godoi, I. P., Lemos, L. L., de Araujo, V. E., Bonoto, B. C., Godman, B., and Guerra Junior, A. A. (2017). CYD-TDV dengue vaccine: systematic review and metaanalysis of efficacy, immunogenicity and safety. J. Comp. Eff. Res. 6, 165-180. doi: 10.2217/cer-2016-0045

Goncalvez, A. P., Engle, R. E., St Claire, M., Purcell, R. H., and Lai, C. J. (2007). Monoclonal antibody-mediated enhancement of dengue virus infection in vitro and in vivo and strategies for prevention. Proc. Natl. Acad. Sci. U.S.A. 104, 9422-9427. doi: 10.1073/pnas.0703498104

Guzman, M. G., Kouri, G., Valdes, L., Bravo, J., Alvarez, M., Vazques, S., et al. (2000). Epidemiologic studies on Dengue in Santiago de Cuba, 1997. Am. J. Epidemiol. 152, 793-799. doi: 10.1093/aje/152.9.793

Halstead, S. B. (1970). Observations related to pathogensis of dengue hemorrhagic fever. VI. Hypotheses and discussion. Yale J. Biol. Med. 42, 350-362.

Halstead, S. B. (1979). In vivo enhancement of dengue virus infection in rhesus monkeys by passively transferred antibody. J. Infect. Dis. 140, 527-533. doi: 10.1093/infdis/140.4.527

Halstead, S. B. (2007). Dengue. Lancet 370, 1644-1652. doi: 10.1016/ S0140-6736(07)61687-0

Halstead, S. B., and O'Rourke, E. J. (1977). Dengue viruses and mononuclear phagocytes. I. Infection enhancement by non-neutralizing antibody. J. Exp. Med. 146, 201-217. doi: 10.1084/jem.146.1.201

Hampe, C. S. (2012). Protective role of anti-idiotypic antibodies in autoimmunity-lessons for type 1 diabetes. Autoimmunity 45, 320-331. doi: 10.3109/08916934.2012.659299

Jessie, K., Fong, M. Y., Devi, S., Lam, S. K., and Wong, K. T. (2004). Localization of dengue virus in naturally infected human tissues, by immunohistochemistry and in situ hybridization. J. Infect. Dis. 189, 1411-1418. doi: 10.1086/383043

John, D. V., Lin, Y. S., and Perng, G. C. (2015). Biomarkers of severe dengue disease - a review. J. Biomed. Sci. 22:83. doi: 10.1186/s12929-015-0191-6

Liao, B., Tang, Y., Hu, F., Zhou, W., Yao, X., Hong, W., et al. (2015). Serum levels of soluble vascular cell adhesion molecules may correlate with the severity of dengue virus-1 infection in adults. Emerg. Microbes Infect. 4:e24. doi: 10.1038/emi.2015.24

Liu, J., Liu, Y., Nie, K., Du, S., Qiu, J., Pang, X., et al. (2016). Flavivirus NS1 protein in infected host sera enhances viral acquisition by mosquitoes. Nat. Microbiol. 1:16087. doi: 10.1038/nmicrobiol.2016.87

Malavige, G. N., Gomes, L., Alles, L., Chang, T., Salimi, M., Fernando, S., et al. (2013). Serum IL-10 as a marker of severe dengue infection. BMC Infect. Dis. 13:341. doi: 10.1186/1471-2334-13-341

Martinez Gomez, J. M., Ong, L. C., Lam, J. H., Binte Aman, S. A., Libau, E. A., Lee, P. X., et al. (2016). Maternal antibody-mediated disease enhancement in type $\mathrm{i}$ interferon-deficient mice leads to lethal disease associated with liver damage. PLoS Negl. Trop. Dis. 10:e0004536. doi: 10.1371/journal.pntd.00 04536 
Ojha, A., Nandi, D., Batra, H., Singhal, R., Annarapu, G. K., Bhattacharyya, S., et al. (2017). Platelet activation determines the severity of thrombocytopenia in dengue infection. Sci. Rep. 7:41697. doi: 10.1038/srep41697

Orozco, S., Schmid, M. A., Parameswaran, P., Lachica, R., Henn, M. R., Beatty, R., et al. (2012). Characterization of a model of lethal dengue virus 2 infection in C57BL/6 mice deficient in the alpha/beta interferon receptor. J. Gen. Virol. 93(Pt 10), 2152-2157. doi: 10.1099/vir.0.045088-0

Osorio, J. E., Velez, I. D., Thomson, C., Lopez, L., Jimenez, A., Haller, A. A., et al. (2014). Safety and immunogenicity of a recombinant live attenuated tetravalent dengue vaccine (DENVax) in flavivirus-naive healthy adults in Colombia: a randomised, placebo-controlled, phase 1 study. Lancet Infect. Dis. 14, 830-838. doi: 10.1016/S1473-3099(14)70811-4

Pan, Y., Yuhasz, S. C., and Amzel, L. M. (1995). Anti-idiotypic antibodies: biological function and structural studies. FASEB J. 9, 43-49.

Ramadhany, R., Hirai, I., Sasaki, T., Ono, K., Ramasoota, P., Ikuta, K., et al. (2015). Antibody with an engineered $\mathrm{Fc}$ region as a therapeutic agent against dengue virus infection. Antiviral Res. 124, 61-68. doi: 10.1016/j.antiviral.2015.10.012

Ramakrishnan, L., Pillai, M. R., and Nair, R. R. (2015). Dengue vaccine development: strategies and challenges. Viral Immunol. 28, 76-84. doi: $10.1089 /$ vim. 2014.0093

Reich, N. G., Shrestha, S., King, A. A., Rohani, P., Lessler, J., Kalayanarooj, S., et al. (2013). Interactions between serotypes of dengue highlight epidemiological impact of cross-immunity. J. R. Soc. Interface 10:20130414. doi: 10.1098/rsif.2013.0414

Rodenhuis-Zybert, I. A., van der Schaar, H. M., da Silva Voorham, J. M., van der Ende-Metselaar, H., Lei, H.-Y., Wilschut, J., et al. (2010). Immature dengue virus: a veiled pathogen? PLoS Pathog 6:e1000718. doi: 10.1371/journal.ppat.1000718

Sangkawibha, N., Rojanasuphot, S., Ahandrik, S., Viriyapongse, S., Jatanasen, S., Salitul, V., et al. (1984). Risk factors in dengue shock syndrome: a prospective epidemiologic study in Rayong, Thailand. I. The 1980 outbreak. Am. J. Epidemiol. 120, 653-669. doi: 10.1093/oxfordjournals.aje.a113932
Screaton, G., Mongkolsapaya, J., Yacoub, S., and Roberts, C. (2015). New insights into the immunopathology and control of dengue virus infection. Nat. Rev. Immunol. 15, 745-759. doi: 10.1038/nri3916

Soo, K. M., Khalid, B., Ching, S. M., and Chee, H. Y. (2016). Metaanalysis of dengue severity during infection by different dengue virus serotypes in primary and secondary infections. PLoS ONE 11:e0154760. doi: 10.1371/journal.pone.0154760

Wang, Y., Si, L. L., Guo, X. L., Cui, G. H., Fang, D. Y., Zhou, J. M., et al. (2017). Substitution of the precursor peptide prevents anti-prM antibody-mediated antibody-dependent enhancement of dengue virus infection. Virus Res. 229, 57-64. doi: 10.1016/j.virusres.2016.12.003

Wichmann, O., Hongsiriwon, S., Bowonwatanuwong, C., Chotivanich, K., Sukthana, Y., and Pukrittayakamee, S. (2004). Risk factors and clinical features associated with severe dengue infection in adults and children during the 2001 epidemic in Chonburi, Thailand. Trop. Med. Int. Health 9, 1022-1029. doi: 10.1111/j.1365-3156.2004.01295.x

Zybert, I. A., van der Ende-Metselaar, H., Wilschut, J., and Smit, J. M. (2008). Functional importance of dengue virus maturation: infectious properties of immature virions. J. Gen. Virol. 89(Pt 12), 3047-3051. doi: 10.1099/vir.0.2008/002535-0

Conflict of Interest Statement: The authors declare that the research was conducted in the absence of any commercial or financial relationships that could be construed as a potential conflict of interest.

Copyright $\odot 2017$ Wang, Yang, Huang, Huang, Zhang, Wang, Zhang and Zhang. This is an open-access article distributed under the terms of the Creative Commons Attribution License (CC BY). The use, distribution or reproduction in other forums is permitted, provided the original author(s) or licensor are credited and that the original publication in this journal is cited, in accordance with accepted academic practice. No use, distribution or reproduction is permitted which does not comply with these terms. 